\title{
Morbilidad Materna, Morbimortalidad Fetal en Diabetes Tipo I y II
}

\author{
Dres. : Gustavo Gómez T., M.D. , Fabio Home M., M.D. ${ }^{* *}$ y Raúl M. Escobar N., M.D.
}

\section{INTRODUCCION}

Se analizan 54 pacientes diabéticas embarazadas en el período comprendido en 1980-1982, controladas en la Clínica de Embarazo de Alto Riesgo del Hospital Universitario del Valle, manejadas en forma conjunta entre el Servicio de Obstetricia y la Clínica de Endocrinología Ginecológica.

Desde 1980 utilizamos una clasificación modificada basada en recomendaciones del Instituto Nacional de Salud de E.U. de A., en Julio de 1979: diabetes tipo I, o insulino dependiente (inicialmente (lamada diabetes de inicio juvenil) y diabetes tipo II o no-insulino dependiente (inicialmente llamada diabetes de inicio en la edad madura); en este último grupo incluímos la diabetes gestacional, anteriormente clase $A$ de la clasificación de White (2). Análisis de morbilidad materna, morbilidad y mortalidad fetal, se enfatizan en este trabajo.

* Profesor Auxiliar. Departamento de Obstetricia y Ginecologia. Coordinador Clínica Endocrinología e Infertilidad. Hospital Universitario del Valle. Cali - Colombia.

* Residente Tercer Año. Departamento de Obstetricia y Ginecología. Hospital Universitario del Valle. Cali - Colombia.

\section{PACIENTES Y METODOS}

Todas las pacientes con riesgo que consultan a la Clínica de Ginecología General, o las pacientes remitidas por centros de referencia de pacientes, son investigadas para detectar lo más temprano posible diabetes complicando el embarazo. Los parámetros por definir pacientes con riesgo han sido descritos anteriormente (3). Si no hay diabetes franca (tipo II) y el embarazo está en el primer o mitad del segundo trimestre, se practica un test de carga con 50 grs. de glucosa, previa glicemia en ayunas. Valores menores de 90 y 140 pre y post carga, respectivamente, se consideran negativos (4). Si el test es positivo se procede a realizar una curva de tolerancia oral a la glucosa (CTOG), con 100 grs. de glucosa y durante 3 horas. Si ésta es negativa se repite la CTOG después de la semana 28 de gestación. En el caso que el test con 50 grs. de glucosa inicial sea negativo, se realiza la CTOG después de la semana 28 de embarazo.

El manejo de la paciente tipo II es a base de dieta, de 30-35 calorías por kilo ideal de peso, para la edad gestacional y el control es mensual, con glucosas pre y post 2 horas, en la clínica de Embarazo de Alto Riesgo (C.E.A.R.). El manejo de la paciente tipo I es a base de dieta más 
insulina $\mathrm{NPH}$, según protocolo publicado anteriormente (3-5).

A partir de la semana 32 se realizaron pruebas de monitoría biofísica fetal anteparto en las diabéticas tipo I y a partir de la semana 34 en las diabéticas tipo II, así: Prueba de No Stress (PNS) y de acuerdo a ésta, posteriormente, prueba de tolerancia a la Ocitocina (PTO).

La PNS se consideró reactiva cuando en un período de 20 minutos había 4 contracciones con incremento $\geqslant 15$ latidos/minuto sobre la línea de base. La PTO positiva implica la aparición de desaceleración tardía en todas las 3 ó 4 contracciones alcanzadas en 10 minutos. Algunas pacientes fueron evaluadas durante contracciones espontáneas.

\section{Ecografía Obstétrica:}

Se realiza para determinar crecimiento fetal intrauterino, tamaño fetal, localización de la placenta y cantidad de líquido amniótico; aunque este examen no estuvo disponible para todas las pacientes, ni en el número de exámenes necesarios. En todos los casos de evacuación electiva se realizó el test de Clements y en casos de duda diagnóstica de madurez pulmonar, falta de correlación entre FUR, test de Clements y examen físico, se practica medición de la relación Lecitina/esfingomielina ( $L / E)$, considerado como indicador de madurez cuando la relación es $\geqslant 2.5$.

\section{Monitoría Fetal}

Se realizó de acuerdo a normas preestablecidas en nuestro protocolo: 32 semanas de gestación en diabéticas Tipo I, 34 semanas en Tipo II, con excepciones de acuerdo a parámetros clínicos o morbilidad materna que indicara la necesidad de iniciarla antes o practicarla más frecuente que semanal, como se hacía en los casos de monitoría normal.

\section{RESULTADOS}

La distribución por edad permanece en los grupos reproductivos más activos de 20 a 30 años con un pico de incremento en el grupo mayor de 35 años (Cuadro No. 1). Como se describió en métodos, todas las pacientes diabéticas tipo II fueron manejadas con dieta. Todas, menos 2 pacientes del grupo tipo I, se manejaron con dieta $+\mathrm{NPH}$. Estas dos pacientes debido a la dificultad para controlar los niveles de glicemia con dosis de NPH; hubo necesidad de utilizar además insulina cristalina (Cuadro No. 2). Una de nuestras muertes fetales sucedió en una de estas pacientes que necesitaron insulina cristalina, con desarrollo de contracciones espontáneas, sufrimiento fetal, detectado por monitoría y muerte intrauterina súbita antes de practicarle cesárea.

Cuadro No. $1^{*}$

\section{DISTRIBUCION POR GRUPOS EDAD}

\begin{tabular}{|ccc|}
\hline \multicolumn{3}{|c|}{ DISTRIBUCION POR GRUPOS EDAD } \\
\hline GRUPOS & $N^{\circ}$ & $\%$ \\
\hline $15-20 \mathrm{a}$. & 4 & 7.4 \\
$21-25 \mathrm{a}$. & 14 & 25.9 \\
$26-30 \mathrm{a}$. & 15 & 26.1 \\
$31-35 \mathrm{a}$. & 9 & 16.6 \\
$>35 \mathrm{a}$. & 12 & 22.2 \\
\hline & 54 & $100 \%$ \\
\hline
\end{tabular}

* Todos los datos estadísticos encontrados en estos cuadros han sido tomados de un estudio de "54 pacientes diabéticas embarazadas," realizado en el Hospital Universitario del ValleDepto. de Ginecología y Obstetricia.

En 15 pacientes se realizaron monitor ías que resultaron patológicas o anormales. (Tabla I) 
Tabla No. 1

MONITORIAS ANORMALES EN 15 DE 54

PACIENTES DIABETICAS EMBARAZADAS

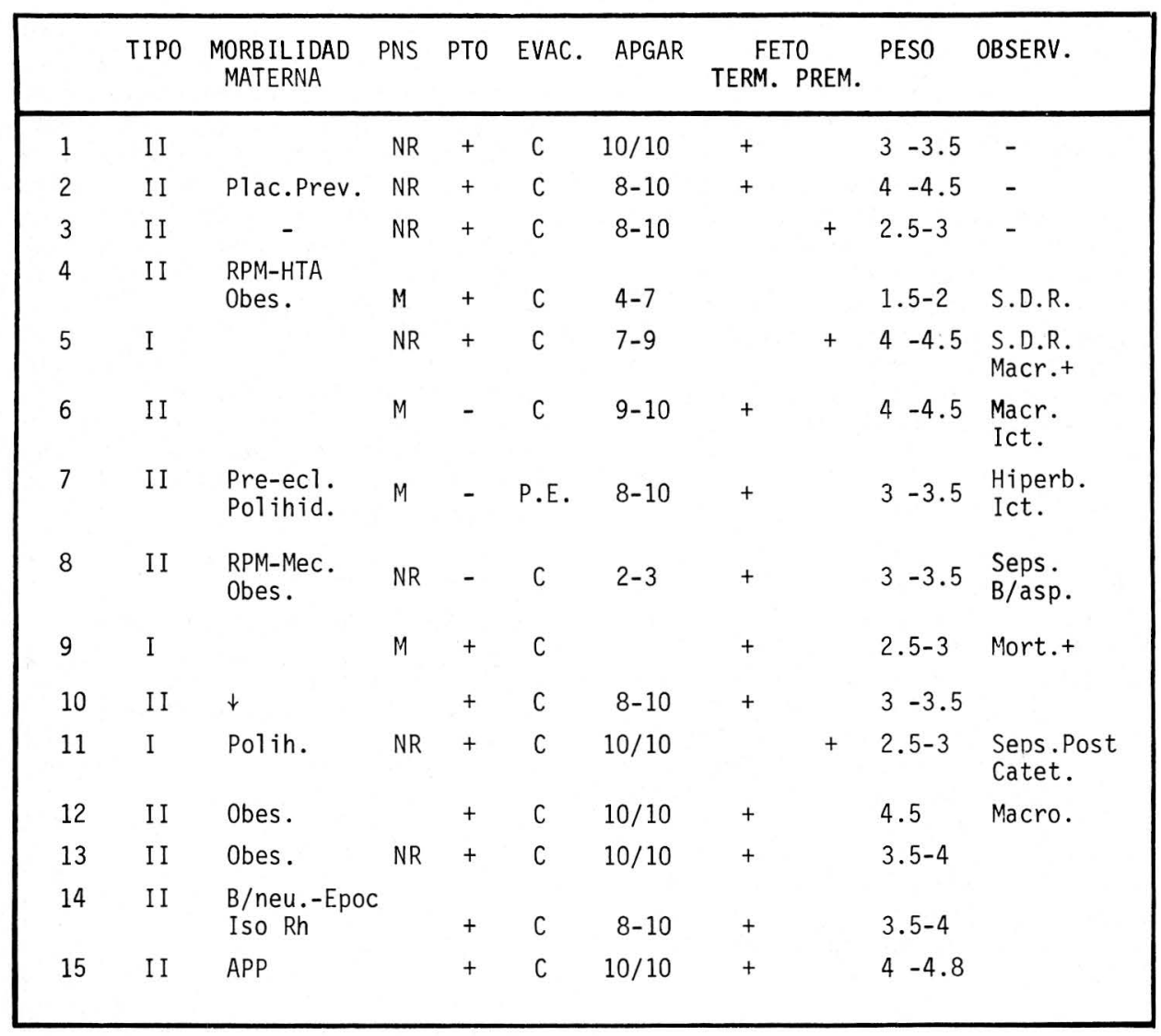

NR : No Reactiva

M : Mixta

+ : PTO con desaceleraciones tardías. Ver texto.

SDR : Sindrome Dificultad Respiratoria.

Macr : Macrosómico.

El $80 \%$ de las monitorías anormales se presentaron en el grupo de diabéticas gestacionales.
Las 3 monitorías anormales en el grupo de diabéticas insulino dependientes terminaron en muertes neonatales; 2 
Cuadro No. 2

\section{TIPO DE TRATAMIENTO}

\begin{tabular}{|c|c|c|}
\hline \multicolumn{3}{|c|}{ TIPO DE TRATAMIENTO } \\
\hline & TIP0 I & TIPO II \\
\hline Dieta solo & 0 & $36 \quad(97 \%)$ \\
\hline Dieta + NPH & $15(88.3 \%)$ & 0 \\
\hline $\begin{array}{c}\text { Dieta }+\mathrm{NPH} \\
+ \\
+ \\
\text { 1. Cristal ina }\end{array}$ & $2(11.7 \%)$ & 0 \\
\hline Ninguno & 0 & $1 \quad(3 \%)$ \\
\hline
\end{tabular}

de estos fetos eran prematuros, aunque en uno de los 2 fetos muertos la defunción fue ocho días post parto, después de desarrollar una septicemia a partir de infección de un pedazo de cateter dejado inadvertidamente por el médico en una venodisección.

De 7 PNS no reactivas, 6 PTO posteriormente fueron positivas. Hubo 4 pruebas de no stress catalogadas como mixtas, en una nomenclatura que se usó durante este período de tiempo, en la clasificación de las monitorías biofísicas, que implica una reactividad cardíaca fetal a algunos movimientos, pero no a más del $50 \%$ de ellos. Dos de estas pruebas tuvieron PTO positivos, otro fue llevado a cesárea por una desproporción feto-pélvica (macrosómico) y el cuarto fue un parto espontáneo pero el feto presenta hiperbilirrubinemia e ictericia neonatal. Lógicamente, todos los fetos que presentaban desaceleraciones en la PTO $(+)$ o ante contractilidad espontánea, terminaron en cesárea (Cuadro No. 5). El apgar de los fetos fue mayor de 8 en todos menos dos casos, uno con PTO (+) y otro únicamente con PNS no reactiva; ambos fetos sobrevivieron.
Se realizaron 40 cesáreas en este grupo de 54 pacientes $(74.1 \%)$. La monitoría fetal anormal, desproporción y meconio, son responsables de las dos terceras partes de las cesáreas (Cuadro No. 5). El apgar al nacer es 7 en el $88.2 \%$ y 83. $7 \%$ en el tipo I y 11 respectivamente (Cuadro No. 3). La edad gestacional valorada por el pediatra al nacer se muestra en el Cuadro 4. Es significantemente mayor el número de prematuros en las pacientes tipo I, con relación al tipo II. La incidencia de macrosómicos en ambos grupos fue estadisticamente igual (Figura No. 1).

Cuadro No. 3

54 PACIENTES DIABETICAS EMBARAZADAS APGAR FETAL 10 MINUTOS

\begin{tabular}{|llllll|}
\hline & & $0-5$ & $5-7$ & $>7$ \\
\hline TIPO & I & $(17)$ & $1(5.8)$ & $1(5.8)$ & $15(88.2)$ \\
TIPO & II & $(37)$ & $3(8.1)$ & $3(8.1)$ & $31(83.7)$ \\
\hline
\end{tabular}

Cuadro No. 4

54 PACIENTES DIABETICAS EMBARAZADAS EDAD GESTACIONAL

\begin{tabular}{|lllllll|}
\hline & & A TERMINO & PREMATURO \\
\hline TIPO & I & $(17)$ & 11 & $(64.6)$ & 6 & $(35.2)$ \\
TIPO & II & $(37)$ & 34 & $(91.9)$ & 3 & $(8.1)$ \\
\hline
\end{tabular}

La morbilidad materna se muestra en la tabla No. 3. La cifra más significativa es la prematurez, que como lo habíamos mencionado antes, es cuatro veces mayor en las diabéticas tipo I que las de tipo II. La macrosomía es ligeramente mas elevada en las pacientes del tipo I. El resto de morbilidad no muestra diferencias entre ambos tipos. 
Cuadro No. 5

54 PACIENTES DIABETICAS EMBARAZADAS CAUSA DE CESAREA

\begin{tabular}{|c|c|c|}
\hline Monitoria Anormal & 14 & $-35 \%$ \\
\hline Desproporción F.P. & 10 & $-25 \%$ \\
\hline Meconio & 5 & $-12.5 \%$ \\
\hline Distocia Pélvica & 3 & \\
\hline Toxemia & 2 & \\
\hline Inducciōn Fallida & 3 & \\
\hline Cesárea Previa & 1 & \\
\hline Placenta Previa & 1 & \\
\hline Distocia Cervical & 1 & \\
\hline TOTAL & 40 & $-74.1 \%$ \\
\hline
\end{tabular}

La mortalidad perinatal en este grupo (4/54) $7.4 \%$, muestra estas caracteristicas: a) tres tenían pruebas anteparto, PNS y PTO anormales, (casos 5-9-11, tabla No. 1). El caso 5 nace con apgar $7 / 9$, para 4.300 grs, es catalogado como prematuro, muere por S.D.R. severa. El caso 9 hace una PNS mixta con posterior PTO + ; se demora 10 horas en operar esta paciente y el niño muere in útero. En el caso 11 es posiblemente el más previsible de todos: nace feto con apgar $10 / 10$ pero entre $2.500-3.000$ grs, muere ocho días después por sepsis a partir de un pedazo de cateter de polietileno que se queda al retirar la venodisección umbilical. La cuarta muerte fetal está relacionada con morbilidad materna concomitante; es una diabética tipo II que presenta R.P.M. y llega con amnionitis, bronconeumonía, desarrolla una toxemia severa y presenta parto espontáneo de feto prematuro, apgar $3 / 4$, que muere en las primeras 48 horas de vida.

\section{COMENTARIOS}

En el manejo de la paciente diabética embarazada se pone de manifiesto la dis-

Figura No. 1

PESO AL NACER. 54 PACIENTES DIABETICAS EMBARAZADAS

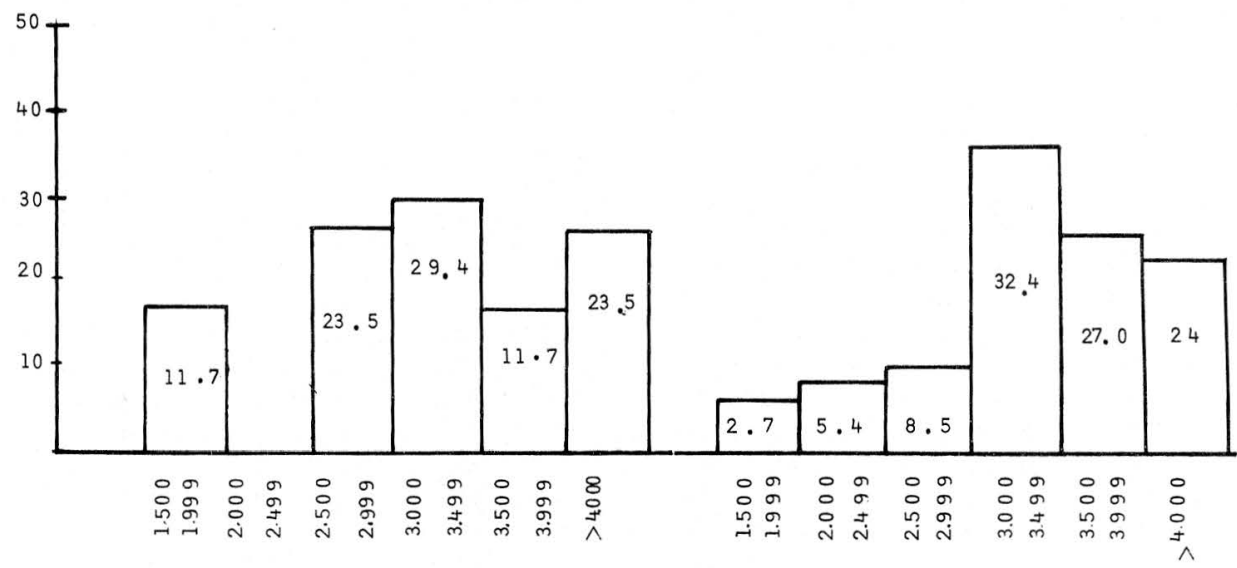

Tipo I

Tipo II 
minución de la morbilidad y mortalidad neonatal, si se logran mantener los niveles de glicemia en valores cercanos a los normales en mujeres embarazadas no diabéticas. En estas pacientes los niveles de glicemia en ayunas promedian $65-88 \mathrm{mg}$ /dl y post prandial de 90-120 mg/dl; Karlson y Kjellmer encontraron en 1972 que la mortalidad perinatal de $24 \%$ en pacientes con niveles de glucosa mayor de $150 \mathrm{mg} / \mathrm{dl}$, se reduce a $3.6 \%$ cuando los niveles de glucosa se mantienen por debajo de $100 \mathrm{mg} / \mathrm{dl}$ (7). Desde entonces han proliferado las publicaciones en que la mortalidad baja al $5 \%$ cuando se hace un estricto control de glicemia (8-9-10). La morbilidad fetal es tal que se han reportado malformaciones congénitas en 5 a 18 por ciento de casos (11-12), macrosom ía en 16 a 40 por ciento (12-13), hipoglicemia de 8-22 por ciento (12-13); hiperbilirrubina e ictericia en 19-35 por ciento (12-13-14) y sindrome de dificultad respiratoria en 2 a 9 por ciento de los casos estudiados (12-13). La mortalidad fetal ha variado en series reportadas en últimos 10 años de $0-20$ por ciento (7-15).

La mortalidad global no corregida en este estudio fue del $7.4 \%$, que comparada con la reportada en la literatura inglesa $(0.21 \%)$, está en niveles inferiores. $\mathrm{EI}$ grupo mejicano de Zárate y colaboradores informan una mortalidad perinatal global en un grupo de 106 embarazadas diabéticas del $11 \%$. Es de anotar que dos muertes perinatales fueron por causas posiblemente no debidas al estado diabético en sí y esto daría una mortalidad perinatal corregida de $3.7 \%$ en este grupo de pacientes. Comparado con la mortalidad perinatal global en el servicio de Ginecología y Obstetricia del H.U.V., la mortalidad perinatal "no corregida", en nuestras pacientes diabéticas es ligeramente mayor. No hubo malformaciones congénitas detectables al nacimiento, en este estudio.

A pesar de que el manejo prenatal de las pacientes diabéticas ha reducido la
Tabla No. 2

MORBILIDAD MATERNA EN 54 PACIENTES DIABETICAS EMBARAZADAS

\begin{tabular}{|lcc|}
\hline & TIPO II (37) & TIPO I (17) \\
\hline Obesidad & 13.5 & 5.8 \\
Toxemia & 8.1 & 11.7 \\
R.P.M. & 10.8 & - \\
HTA & 13.5 & 11.7 \\
Polihidramnios & 5.4 & - \\
\hline
\end{tabular}

mayoría de la morbilidad, lo mismo que la mortalidad fetal, la incidencia de malformaciones congénitas permanece inalterada en los últimos 25 años (13). Goldman y colaboradores, usando un enfoque de desarrollo morfológico para determinar cuál fue el desarrollo fetal en el momento de iniciar una determinada malformación congénita, concluyó que éstas ocurren siempre antes de la séptima semana de gestación (16). El diagnóstico y manejo antes de la semana 12 manteniendo niveles de glicemia hasta el momento del parto en $\pm 80 \mathrm{mg} / \mathrm{dl}$, comparado con un grupo control no diabético, no se encuentra mayor incidencia de

Tabla No. 3

\section{MORBIMORTALIDAD FETAL EN 54 DIABETICAS EMBARAZADAS}

\begin{tabular}{|lcc|}
\hline & TIPO II (37) & TIPO I (17) \\
\hline . Prematurez & $8.1 \%$ & $35.2 \%$ \\
. Macrosomía & 10.8 & 17.6 \\
. Hipoglicernia & 5.4 & 5.8 \\
. Bajo peso para Edad Gestacional & 5.4 & 11.7 \\
. S.D.R. & 8.1 & 5.8 \\
. Ictericia & 5.4 & 5.8 \\
Muertes Perinatales & $2.7^{\star}$ & 11.7 \\
Mortinatos & - & 5.8 \\
\hline
\end{tabular}

* Mortalidad Neonatal secundaria a RPM, Amnionitis, Prematuro. 
morbilidad incluyendo malformaciones congénitas, en el grupo diabético (17). En lo anterior radica la importancia de que en las pacientes de alto riesgo que desarrollen diabetes durante el embarazo, sea ésta detectada lo más temprano posible durante la gestación. Tanto las diabéticas tipo I -ya conocidas como diabéticas antes de embarazarse-, como las diabéticas tipo II -gestacional-, deben mantener desde antes de la séptima semana de gestación, valores < de $90 \mathrm{mg} / \mathrm{dl}$ en ayunas $\mathrm{y} \leqslant 140 \mathrm{mg} / \mathrm{dl}$ a la hora postprandial.

\section{BIBLIOGRAFIA}

1. FELIG, P., COUSTAN, D. Diabetes Mellitus in Syllabus intensive review course in reproductive endocrinology. Harvard Medical School. 1982.

2. WHITE, P. Pregnancy complicating Diabetes. Am. J. Med. 7:609, 1949.

3. GOMEZ, G., BRITTO, R. Diabetes y Embarazo. Rev. Col. Obstet. Gynecol. 31: $104,1980$.

4. O'Sullivan, J.B. Establishing criteria for gestational diabetes. Diabetes Care 3:437, 1980.

5. GOMEZ, G. Diabetes y Embarazo. Procolo de Manejo, 1983.

6. COUSINS, L., RIGG, L., HOLLINGSWORTH, D., BRINK, G., AVRAND, J. and YEN S.S.C. The 24 hours excursion and diurnal rhythm of glucose, insulineand c. peptide in normal pregnancy. Am. J. Obstet. Gynecol. 136:483, 1980.

7. KARLSON, K., K. JELLMER I. The outcome of diabetic pregnancy in relation to the mother blood sugar levels. Am. J. Obstet. Gynecol. 112:213, 1972.

8. COUSTAN, D.R., BERKOWITZ, R.L. and HUBBINS, J.C. Tight metabolic control of overt diabetes in pregnancy. Am. J. Obstet. Gynecol. 68:845, 1980.
La morbilidad materna (tabla No. 2), mostró que la hipertensión arterial y la toxemia tienen una incidencia similar en ambos grupos. La obesidad, RPM y polihidramnios se presentan en una proporción mayor en la diabetes tipo II. Esto sugiere que en la diabetes gestacional deben controlarse y monitorizarse madre y feto tan estrictamente como en la diabetes tipo I, para prevenir morbilidad materna y fetal, e inclusive mortalidad fetal.

9. GABBE, S.G., MESTMAN, J.H., FREEMAN, R., ANDERSON, G., LOWENSOHN. Management and outcome of close A Diabetes mellitus. Am. J. Obstet. Gynecol. 127:465, 1977.

10. GABBE, S.G., MESTMAN, J.H., FREEMAN, R., GOEBELSMANN, U.T., LOWENSOHN, R., NOCHIMSON, D., CETRULLO C. and QUILLIGAN, E. Management and outcome in diabetes mellitus classes B to R. Am. J. Obstet. Gynecol. 129:723, 1977.

11. KUCERA, T. Rate and type of congenital anomalies among offspring of diabetics women. J. Reprod. Med. 7: 61, 1970.

12. KITZMILLER, J.L., CLOHERTY, J.P. YOUNGER, M.D., et. al. Diabetic Pregnancy and perinatal morbility. Am. J. Obstet. Gynecol. 131:560, 1978.

13. SOLER, N.G., SOLER, S.M., MALINS, J.M. Neonatal morbility among infants of diabetic mothers. Diabetes Care 1:340, 1978.

14. WHITE, P. Diabetes Mellitus in Pregnancy. Clin. Perinatal 1:331, 1974.

15. ADASHI, E.Y., PINTO, H., TYSON, J.E. Impact of maternal euglycemia on fetal outcome in diabetic pregnancy. Am. J. Obstet. Gynecol. 133:268, 1979. 
16. MILLS, J., BAKER, L., GOLDMAN, A. Malformation in infants of diabetic mothers occur before the seventh gestational week: Implication for treatment. Diabetes 28:292, 1979.

17. JOVANOVICS, L., DRUZIN, M., PETERSON, CH. Effects of euglycemia on the outcome of pregnancy in insulin-dependent diabetic women as compared with normal control subjects. Am. J. Med. 7:921, 1981.

18. ZARATE, A., CANALES, E., CANO, C., VILLALOBOS, M., PANIAGUA, H, CASTELO, I. Manejo de la diabética embarazada en un Hospital de Ginecobs. tetricia. Rev. Invest. Clin. (Mex.) 34:205, 1982. 